\title{
Hemotórax masivo tardío por transección de la arteria pericardiofrénica por fractura costal. Reporte de un caso
}

\author{
Late massive hemothorax by transection of the pericardiophrenic artery by costal \\ fracture. Report of a case
}

\author{
Daniel A. Montalvo-López ${ }^{1 *}$, José López-Reséndiz y Javier García-Álvarez²

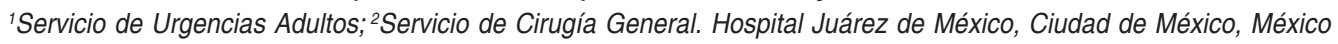

\begin{abstract}
Resumen
Hasta un $8 \%$ de los pacientes sometidos a toracotomía urgente tras un traumatismo cerrado presentan una lesión costal que había pasado inadvertida inicialmente. Tal es el caso clínico que presentamos, en el que la lesión costal pasó inadvertida en la evaluación inicial y 72 horas después, al referir el paciente dolor en el hemitórax izquierdo, se solicitó tomografía simple y contrastada de tórax, y se evidenció hemotórax masivo y fracturas de arcos costales $5 .^{\circ}$ y $6 .^{\circ}$ izquierdos en su porción lateral, además de fractura de los arcos costales $7 .^{\circ}$ y $8 .^{\circ}$ en su porción paraesternal. Se realizó toracotomía exploradora con reparación diafragmática y ligadura de arteria.
\end{abstract}

Palabras clave: Arteria pericardiofrénica. Hemotórax. Traumatismo cerrado de tórax.

\begin{abstract}
Up to $8 \%$ of patients undergoing urgent thoracotomy after closed trauma present a rib lesion that had initially gone unnoticed, such is the clinical case that we present, passing the inadvertent costal lesion in the initial evaluation, 72 hours later refers to pain level of left hemithorax, simple and contrasted chest tomography was requested, showing massive hemothorax and fractures of left costal arches in its lateral portion of $5^{\text {th }}$ and $6^{\text {th }}$, in addition to fracture of the $7^{\text {th }}$ and $8^{\text {th }}$ costal arch in its parasternal portion, exploratory thoracotomy was performed with diaphragmatic repair and artery ligation
\end{abstract}

Key words: Pericardiofrenic artery. Hemothorax. Closed chest trauma.

\section{Introducción}

Los politraumatismos constituyen un serio problema de salud pública, en términos de mortalidad, pues son la primera causa de fallecimiento en las personas menores de 40 años. El traumatismo cerrado de la pared torácica representa el $15 \%$ de todos los ingresos por traumatismos a los departamentos de emergencias en todo el mundo'.

La incidencia estimada de lesión diafragmática en pacientes ingresados de urgencia por traumatismo cerrado toracoabdominal es del $5 \%$, y hasta un $50 \%$ de los casos pasan desapercibidos al ingreso. Tan solo el $15 \%$ de los traumatismos cerrados llegan a requerir

\section{Correspondencia:}

*Daniel A. Montalvo-López

Antares, 29

Fecha de recepción: 29-01-2020 C.P. 02100, Ciudad de México, México

E-mail: danomontalvo@ hotmail.com
Cir Cir. 2020;88(S1):124-128

Contents available at PubMed www.cirugiaycirujanos.com 0009-7411/@ 2020 Academia Mexicana de Cirugía. Publicado por Permanyer. Este es un artículo open access bajo la licencia CC BY-NC-ND (http://creativecommons.org/licenses/by-nc-nd/4.0/). 
cirugía urgente, de los cuales el 8\% presentan lesión diafragmática que había pasado inadvertida inicialmente y son sometidos a toracotomía urgente. Los pacientes con trauma cerrado de tórax pueden ser difíciles de pronosticar en el servicio de urgencias debido a la aparición frecuente de complicaciones tardías².

El hemotórax masivo de tipo traumático es potencialmente mortal, y se acompaña de fracturas de arcos costales hasta en un $10 \%$ de los casos. El impacto de esta lesión es significativo, pues se ha observado que más del $80 \%$ de los pacientes con trauma torácico cerrado que se acompaña de hemotórax requieren ingreso en la unidad de cuidados intensivos y casi el $60 \%$ requerirá ventilación mecánica, con una tasa de traqueotomía del $20 \%$. El hemotórax masivo tardío después de un traumatismo torácico es infrecuente ${ }^{2,3}$.

Presentamos un caso de esta situación poco frecuente con hemotórax masivo tardío causado por una fractura espiculada de borde costal.

\section{Caso clínico}

Varón de 31 años previamente sano sin antecedentes de importancia, que refirió haber iniciado su padecimiento 3 días previos a su ingreso al servicio de urgencias de adultos, secundario a una contusión torácica por caída de motocicleta a baja velocidad con lesiones en el hombro, la cara lateral izquierda y anterior del tórax, y el área precordial (área de Murdock) por el manubrio de la motocicleta, presentando de manera inmediata dolor de tipo punzante, de intensidad $7 / 10$, el cual se exacerba con la inspiración profunda. En la exploración física se encontró presión arterial de 110/70 $\mathrm{mmHg}$, frecuencia cardiaca de $86 \mathrm{lpm}$, frecuencia respiratoria de $21 \mathrm{rpm}$, temperatura de $36.5^{\circ} \mathrm{C}$ y saturación de oxígeno del 95\%, sin alteraciones neurológicas, tórax normolíneo, doloroso a la palpación en hemitórax izquierdo de predomino en los arcos costales $5 .^{\circ}$ y $6 .^{\circ}$, sin crepitación ósea, campos pulmonares con adecuada entrada y salida de aire, no estertores ni sibilancias, claro pulmonar a la percusión, no se integra síndrome pleuropulmonar, ruidos cardiacos rítmicos de buen tono e intensidad, abdomen blando depresible, peristasis presentes, no doloroso a la palpación, sin datos de irritación peritoneal. Por lo anterior se manejó con analgésico por vía intravenosa y se solicitó radiografía de tórax en proyección anteroposterior (AP), en la que no se detectó la presencia de fracturas costales, consolidaciones, colecciones ni signos de neumotórax, motivo por el cual se decide su egreso del servicio con datos de alarma y cita abierta a urgencias (no se solicitó radiografía lateral de tórax). Pasadas 72 horas, el paciente ingresó nuevamente al servicio de urgencias con dolor de tipo punzante en el hemitórax izquierdo en el área precordial, de tipo opresivo, secundario a maniobra de Valsalva, de intensidad 9/10, que se exacerba con el movimiento y el esfuerzo, acompañándose de disnea moderada y diaforesis (Fig. 1). Se encontró alerta, tórax con disminución de los movimientos de amplexión y amplexación, notándose un aumento de la mecánica ventilatoria, hipoventilación en el hemitórax izquierdo sin estertores ni sibilancias, así como respuesta inicial de tipo hipertensora al choque hipovolémico, con presión arterial de 170/100 mmHg, frecuencia cardiaca de $120 \mathrm{lpm}$, frecuencia respiratoria de $30 \mathrm{rpm}$ y saturación de oxígeno del 91\%, agregándose palidez generalizada de tegumentos, por lo que se solicitan paraclínicos de control que reportan potasio $3.7 \mathrm{mEq} / \mathrm{l}$, sodio $139 \mathrm{mEq} / \mathrm{l}$, creatinina $0.78 \mathrm{mg} / \mathrm{dl}$, nitrógeno ureico $23 \mathrm{mg} / \mathrm{dl}$, urea $49 \mathrm{mg} / \mathrm{dl}$, leucocitos $13.8 \mathrm{u} / \mathrm{l}$, hemoglobina $15.5 \mathrm{~g} / \mathrm{dl}$, hematocrito $48.2 \%$ y plaquetas 361.000 u/l. Se solicitó tomografía simple y contrastada de tórax y abdomen, que evidenció hemotórax masivo y fracturas espiculadas de los arcos costales izquierdos $5 .^{\circ} \mathrm{y}$ $6{ }^{\circ}$ en su porción lateral, además de fractura de los arcos costales $7 .^{\circ}$ y $8 .^{\circ}$ en su porción paraesternal (Figs. 2 y 3 ). Se determinó realizar la intervención quirúrgica de urgencia por parte del servicio de cirugía cardiotorácica, así como manejo avanzado de la vía aérea y reanimación con cristaloides, realizándose toracotomía exploradora con reparación diafragmática y ligadura de la arteria pericardiofrénica, obteniendo $2.500 \mathrm{ml}$ de sangrado. Se encontró lesión diafragmática ocasionada por el borde costal de la fractura espiculada. Se realizó ligadura de la arteria y resección de un fragmento de la $6 .{ }^{a}$ costilla, y se colocaron dos sondas endopleurales, una anterior y una posterior (Fig. 4). En controles posteriores de laboratorio se observó un descenso de la hemoglobina de $3 \mathrm{~g} / \mathrm{dl}$. El paciente se mantuvo estable durante la cirugía, requiriendo transfusión de tres concentrados eritrocitarios y tres unidades de plasma fresco congelado. Ameritó ingresó por 72 horas a la unidad de ciudad intensivos, donde se encontró estable hemodinámicamente, y luego pasó a piso de cirugía general, donde continuó con buena evolución hasta que fue dado de alta. Se revaloró un mes después, sin registrarse complicación alguna.

\section{Discusión}

La arteria pericardiofrénica es una rama de la arteria torácica interna que va hacia el diafragma, donde 
se anastomosa con las arterias musculofrénicas y frénicas superiores, discurre con el nervio frénico entre la pleura y el pericardio, y suministra sangre al pericardio y el diafragma ${ }^{4}$.

El trauma cerrado de tórax se define como una lesión torácica que resulta de una contusión de la pared torácica o de fracturas de costillas, con o sin lesión pulmonar no inmediata que pone en peligro la vida. Los arcos costales más comúnmente fracturados son del $5 .^{\circ}$ al $10 .^{\circ}$, con independencia de la cinemática del trauma ${ }^{5}$.

Cuando se observan fracturas de la $1 .^{-}$a la $4 .^{\text {a }}$ costilla, se asocia con lesiones nerviosas o vasculares. Las fracturas de los arcos costales 10. a 12. a menudo se asocian con lesiones en la cavidad abdominal, incluidos el bazo, el hígado y el retroperitoneo. Aproximadamente el $71 \%$ de los casos de hemotórax masivo tardío aparecen descritos en la literatura siempre asociados a traumatismos costales bajos desplazados ${ }^{6,7}$.

Como criterios de gravedad se recomienda considerar la fractura de más de dos costillas, especialmente para pacientes mayores de 65 años, con enfermedades cardiopulmonares, enfermedades de la coagulación o tratamientos anticoagulantes o antiplaquetarios previos, o insuficiencia circulatoria (presión arterial sistólica $<110 \mathrm{mmHg}$ o más de un $30 \%$ de disminución), aumentando la mortalidad en dos a tres veces de lo habitual si el paciente presenta alguno de estos factores de riesgo (recomendación $1 \mathrm{~A})^{8,9}$.

El hemotórax masivo es una afección potencialmente mortal que se asocia con una alta tasa de mortalidad y requiere una intervención clínica y quirúrgica de manera inmediata. El hemotórax masivo consiste en el acúmulo súbito de más de $1.500 \mathrm{ml}$ de sangre o de un tercio de la volemia del paciente en la cavidad pleural, siendo de manera frecuente resultado de lesiones de la vasculatura pulmonar, los grandes vasos o el corazón. El hemotórax tardío resultante de un traumatismo torácico cerrado puede desarrollarse en un plazo de 3 a 6 horas, pero puede retrasarse hasta 30 días, aunque la mayoría de los pacientes (86\%) se diagnostican dentro de los primeros 4 días ${ }^{3,10}$.

El cuadro clínico de un hemotórax masivo tardío puede incluir dolor de tipo pleurítico, diaforesis, ansiedad y principalmente disnea de manera súbita, encontrando en la exploración física datos francos de choque hipovolémico, tales como hipotensión arterial sistémica, palidez de piel y tegumentos, piel fría, taquicardia y taquipnea ${ }^{11,12}$.

Mas allá de la exploración física, a todos los pacientes con trauma de tórax se les debe realizar una

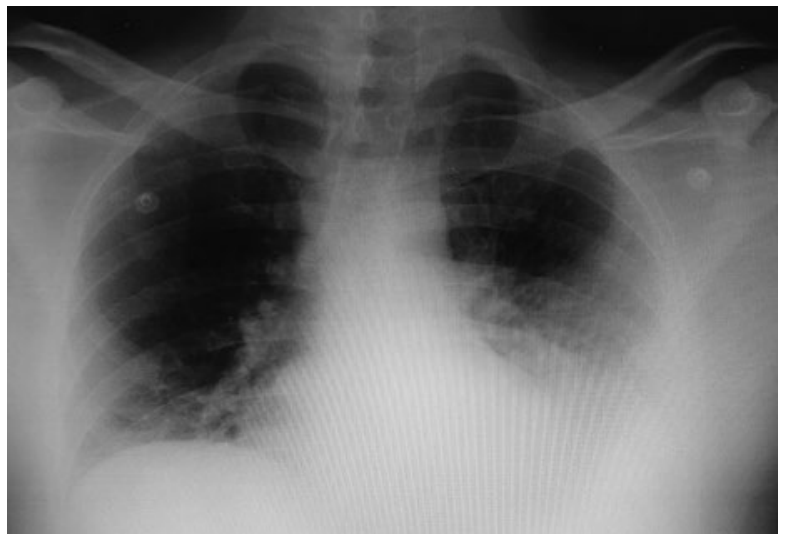

Figura 1. Radiografía de tórax en proyección anteroposterior en la que se evidencian signos de hemotórax izquierdo.

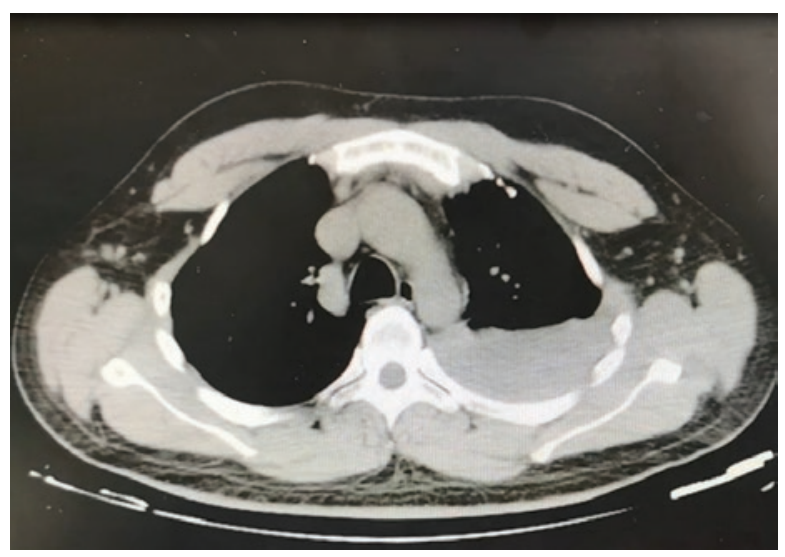

Figura 2. Tomografía computada simple y contrastada de tórax en la que se visualiza el hemotórax izquierdo.

radiografía (proyección posteroanterior y lateral en pacientes estables, y AP en pacientes inestables) como parte de la evaluación inicial; sin embargo, la sensibilidad de la radiografía de tórax para fracturas costales se considera baja (15\%), variando según la ubicación de la fractura ${ }^{12,13}$.

En el servicio de urgencias, la evaluación dirigida con ecografía para el paciente hemodinámicamente inestable se encontró superior a la radiografía de tórax para el diagnóstico de neumotórax, hemotórax y derrame pleural, con una sensibilidad del $78,6 \%$ una especificidad del $98,4 \%$. Si el paciente se encuentra hemodinámicamente estable se recomienda el uso de tomografía simple y contrastada, siendo el método de referencia para evaluar exhaustivamente las lesiones postraumáticas ${ }^{7,8,14}$.

Una vez establecido el diagnóstico, el manejo inicial consiste en la rápida reposición de volumen y la descompresión de la cavidad torácica mediante drenaje 


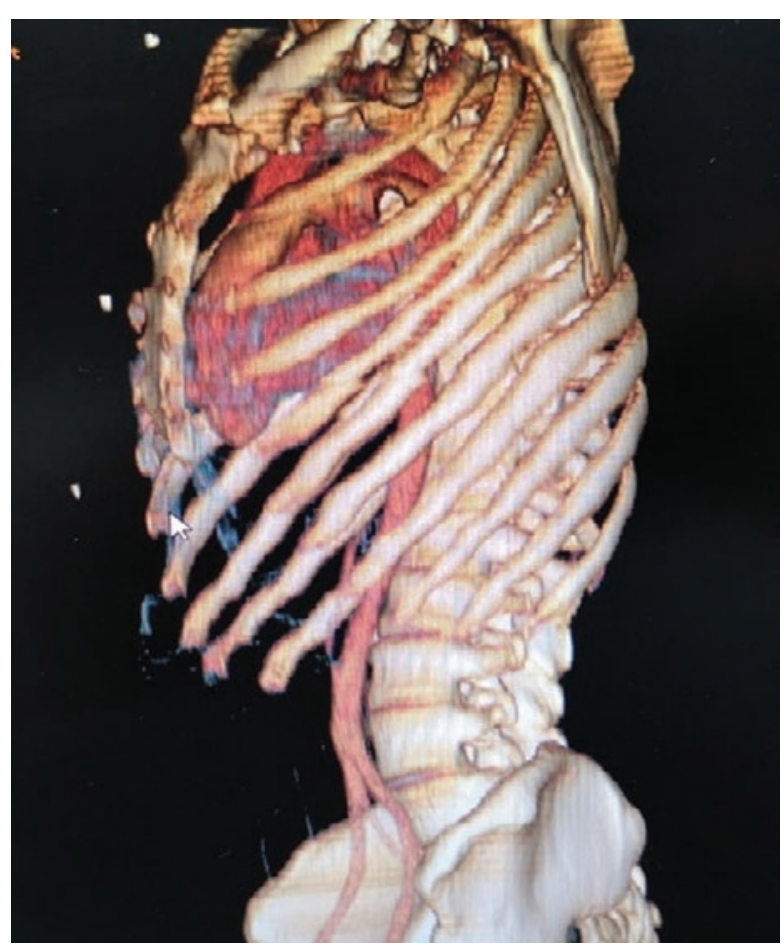

Figura 3. Reconstrucción tridimensional de tomografía computada de tórax que muestra la fractura de los arcos costales izquierdos $5 .^{\circ}$ y $6 .^{\circ}$.

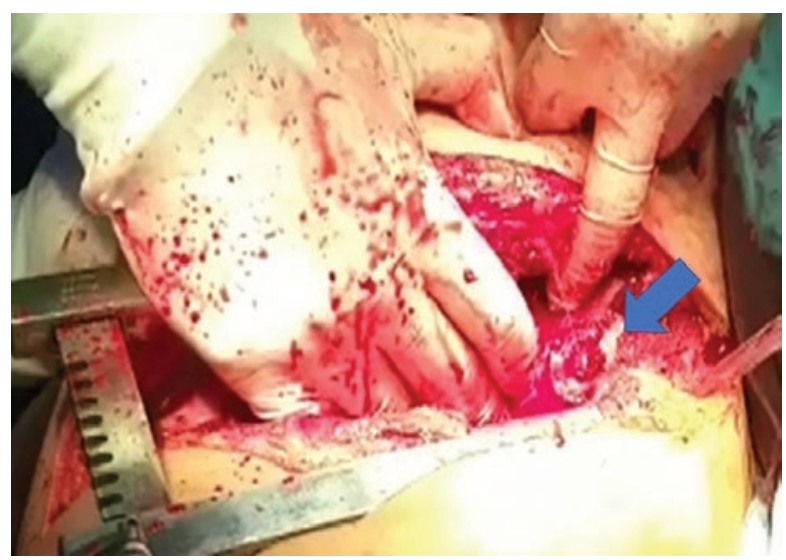

Figura 4. Toracotomía exploradora con reparación diafragmática y ligadura de la arteria pericardiofrénica.

endotorácico. Se han descrito tres tiempos en los que se puede llevar a cabo la intervención dependiendo del estado hemodinámico del paciente: 1) inmediato (toracotomía en la sala de urgencias), para lesiones cardiacas compensadas, lesiones de grandes vasos (aorta), lesiones traqueobronquiales y lesiones esofágicas; 2) urgente (en la sala de quirófano, dentro de 1 a 4 horas de la llegada); y 3) retrasado (24 horas después de la admisión) $)^{12,15}$.
Una vez tomada la decisión de colocar una sonda endopleural, el drenaje de sangre de más de $1.500 \mathrm{ml}$ o de $200 \mathrm{ml} / \mathrm{h}$ durante 2-4 horas, con independencia del volumen inicialmente evacuado, sugiere la presencia de una lesión vascular mayor que es poco probable que se detenga sin intervención quirúrgica, haciendo que la exploración torácica se vuelva obligatoria ${ }^{1,16,17}$.

En relación con el control del dolor intenso en estos pacientes, después de 12 horas de tratamiento analgésico apropiado o en pacientes con riesgo de desarrollo de complicaciones respiratorias (como más de cuatro costillas fracturadas, mayores de 45 años, esfuerzo respiratorio insuficiente) se debe realizar anestesia locorregional de manera inicial o bien analgesia epidural para lesiones compleja o bilaterales ${ }^{8,18}$.

La anestesia regional consiste en bloqueos nerviosos intercostales, bloqueos paravertebrales y catéteres epidurales torácicos. Para garantizar un bloqueo adecuado y evitar la falta de nervios superpuestos inervadores, los bloqueos intercostales requieren la inyección a nivel de la costilla fracturada más una costilla arriba y una costilla abajo, típicamente con bupivacaína con o sin opiáceos (fentanilo) ${ }^{3,6}$.

\section{Conclusión}

El trauma de tórax es una situación altamente desafiante en el manejo de urgencias. Requiere conocimientos de las complicaciones que pueden poner en riesgo vital al paciente en pocos minutos, así como de un adecuado manejo primario de las complicaciones que se pueden presentar en el mediano y largo plazo. Es por esto por lo que el manejo de estas lesiones torácicas es esencial en el trabajo de urgencias. Son de suma importancia los antecedentes sobre el mecanismo de lesión. Cuando el paciente se encuentre con inestabilidad hemodinámica, una sola proyección radiográfica de tórax al pie de cama y un estudio ecográfico FAST (Focused Assessment by Sonography in Trauma) dan información suficiente para formular un plan de manejo inicial. Cuando la hemodinamia del paciente lo permita, las radiografías de tórax en dos proyecciones y la tomografía simple y contrastada son de gran utilidad en el manejo. El paciente con trauma torácico requiere estudio y manejo preciso, siempre pensando en la posibilidad de tratamiento quirúrgico que incidirá en su sobrevida.

Se considera de importancia clínica el caso descrito, ya que pasaron desapercibidas las fracturas costales al realizar solo una proyección radiográfica AP de 
tórax en la evaluación inicial, considerando que la lesión diafragmática inadvertida puede ocasionar un hemotórax masivo, especialmente al iniciar la movilización del paciente, quedando enmascarado por el dolor costal de los puntos de fractura. En la literatura no está descrito un hemotórax masivo secundario a transección específica de la arteria pericardiofrénica.

\section{Conflicto de intereses}

Los autores declaran que no existe conflicto de intereses.

\section{Responsabilidades éticas}

Protección de personas y animales. Los autores declaran que para esta investigación no se han realizado experimentos en seres humanos ni en animales.

Confidencialidad de los datos. Los autores declaran que en este artículo no aparecen datos de pacientes.

Derecho a la privacidad y consentimiento informado. Los autores declaran que en este artículo no aparecen datos de pacientes.

\section{Bibliografía}

1. Byun JH, Kim JW, Kang DH, Yang JH, Moon SH, Kim SH, et al. The thorax trauma severity score and the trauma and injury severity score. Medicine (Balt). 2017;96:e8317.
2. Chen $\mathrm{CL}$, Cheng YL. Delayed massive hemothorax complicating simple rib fracture associated with diaphragmatic injury. Am J Emerg Med. 2014;32:818.e3-4.

3. Dennis BM, Bellister SA, Guillamondegui OD. Thoracic trauma. Surg Clin North Am. 2017;97:1047-64.

4. Moore LK. Anatomía con orientación clínica. 8. ${ }^{a}$ ed. Barcelona: Lippincott Williams \& Wilkins; 2018. 1150 p.

5. TLS Advanced Trauma Life Support. American College of Surgeons. 10ma Edición, Chigado USA, 2018. 412-420.

6. Battle CE, Hutchings H, Lovett S, Bouamra O, Jones S, Sen A, et al. Predicting outcomes after blunt chest wall trauma: development and external validation of a new prognostic model. Crit Care. 2014;18:1-8.

7. Senekjian L, Nirula R. Rib fracture fixation: indications and outcomes. Crit Care Clin. 2017;33:153-65.

8. Bouzat P, Raux M, David JS, Tazarourte K, Galinski M, Desmettre T, et al. Chest trauma: first 48 hours management. Anaesth Crit Care Pain Med. 2017;36:135-45.

9. Segura Sampedro JJ, García Gómez F, Arroyo Pareja L, Pardo Prieto SL, Moreno Mata N. Hemotórax masivo tardío por laceración diafragmática asociado a fracturas costales inferiores. Una herida penetrante en el traumatismo cerrado. Cir Esp. 2015;93:e73-5.

10. Tanizaki S, Hayashi H. Massive hemothorax after blunt transverse cervical artery injury. J Emerg Med. 2012;43:e397-9.

11. Wang HC, Chen WL, Yao CT, Chen JH. Delayed hemothorax caused by thymic bleeding after blunt chest trauma. J Emerg Med. 2015;48:77-8.

12. Mattox KL, Moore EE, Feliciano DV, editores. Trauma. 7th ed. New York: McGraw Hill; 2013.

13. Hutchings SD. Trauma and combat critical care in clinical. Springer, Royal Navy and Kings College Hospital; 2016. 529 p.

14. Mazur SM, Pearce A, Alfred S, Goudie A, Sharley P. The F.A.S.T.E.R. trial. Focused assessment by sonography in trauma during emergency retrieval: a feasibility study. Injury. 2008;39:512-8.

15. Muñoz-cruzado VMD, David J, Arredondo P. Manual de algoritmos para el manejo del paciente politraumatizado. Madrid: Asociación Española de Cirujanos; 2018.

16. Jouffroy R, Langeron $\mathrm{O}$, Riou $\mathrm{B}$, Vivien $\mathrm{B}$. Tratamiento del traumatismo grave del adulto en las primeras 24 horas. EMC - Anestesia-Reanimación. 2016;42:1-22.

17. Meredith JW, Hoth JJ. Thoracic trauma: when and how to intervene. Surg Clin North Am. 2007;87:95-118.

18. Powell BS, Magnotti LJ, Schroeppel TJ, Finnell CW, Savage SA, Fischer PE, et al. Diagnostic laparoscopy for the evaluation of occult diaphragmatic injury following penetrating thoracoabdominal trauma. Injury. 2008;39:530-4. 